\title{
Investigation of Range Profiles from a Simplified Ship on Rough Sea Surface and Its Multipath Imaging Mechanisms
}

\author{
Siyuan He, ${ }^{1}$ Fan Zhang,, ${ }^{1}$ Weidong Hu, ${ }^{2}$ Lei Zhuang, ${ }^{3}$ Xingbin Ye, ${ }^{3}$ and Guoqiang Zhu ${ }^{1}$ \\ ${ }^{1}$ School of Electronic Information, Wuhan University, Wuhan 430079, China \\ ${ }^{2}$ ATR Laboratory, National University of Defense Technology, Changsha 410073, China \\ ${ }^{3}$ Satellite General Department, Shanghai Institute of Satellite Engineering, Shanghai 200240, China
}

Correspondence should be addressed to Siyuan He, siyuanhi@gmail.com

Received 4 August 2011; Revised 15 November 2011; Accepted 5 January 2012

Academic Editor: Ananda Sanagavarapu Mohan

Copyright (C 2012 Siyuan He et al. This is an open access article distributed under the Creative Commons Attribution License, which permits unrestricted use, distribution, and reproduction in any medium, provided the original work is properly cited.

\begin{abstract}
The range profiles of a two-dimension (2 D) perfect electric conductor (PEC) ship on a wind-driven rough sea surface are derived by performing an inverse discrete Fourier transform (IDFT) on the wide band backscattered field. The rough sea surface is assuming to be a PEC surface. The back scattered field is computed based on EM numerical simulation when the frequencies are sampled between $100 \mathrm{MHz}$ and $700 \mathrm{MHz}$. Considering the strong coupling interactions between the ship and sea, the complicated multipath effect to the range profile characteristics is fully analyzed based on the multipath imaging mechanisms. The coupling mechanisms could be explained by means of ray theory prediction and numerical extraction of the coupling currents. The comparison of the range profile locations between ray theory prediction and surface current simulation is implemented and analyzed in this paper. Finally, the influence of different sea states on the radar target signatures has been examined and discussed.
\end{abstract}

\section{Introduction}

The radar target signatures of a ship or target under the marine environment have been an attractive subject in electromagnetic field because it has many applications on radar surveillance, remote sensing, automatic target recognition (ATR), and so forth [1-3]. In recent years, many numerical methods have been developed for the composite scattering of target and rough surface models, for example, the forward backward method with spectral acceleration algorithm (GFBM/SAA) [4], the bidirectional analytic ray tracing (BART) method [5], the extended propagation-insidelayer expansion combined with the generalized-forwardbackward (EPILE + GFBM) method [6], the MM-PO hybrid method [7], and the 3DMLUV method [8].

Most previous researches have focused on the study of radar cross-section (RCS) of the target or ship over a rough sea surface. However, little is discussed in the open literature about the wideband radar target signatures of the composite model based on surface current simulation, for example, range profiles, SAR, and ISAR images. The wideband response provides detail information of individual scattering centers within the target's length and a direct measurement of the target's size and shape. The study of wideband signatures for ship at sea involving complex coupling interactions is of great importance to provide reference for ATR. The investigation of this problem is mainly hindered by two difficulties. Firstly, wideband signatures require a much highly efficient algorithm to be employed in the simulation to meet the challenges of large electrical size, multifrequencies samplings, and multirealizations of the rough sea surface. Secondly, the complicated coupling imaging characteristics between ship and sea is extremely difficult to be fully understood and evaluated.

In our previous research, the analysis of one-dimension (1 D) high resolution range profiles (HRRPs) is limited to a simple two-dimension (2 D) square cylinder far above a slightly rough sea surface [9]. When it comes to the realistic oceanic situation, the model of a ship on sea surface is of much more practical importance. Compared with the target set at a certain height above sea surface [9], the ship right on the sea leads to stronger coupling interactions. Furthermore, the ship with hull and deck house results in various coupling multipaths due to the complex geometry on the ship itself 


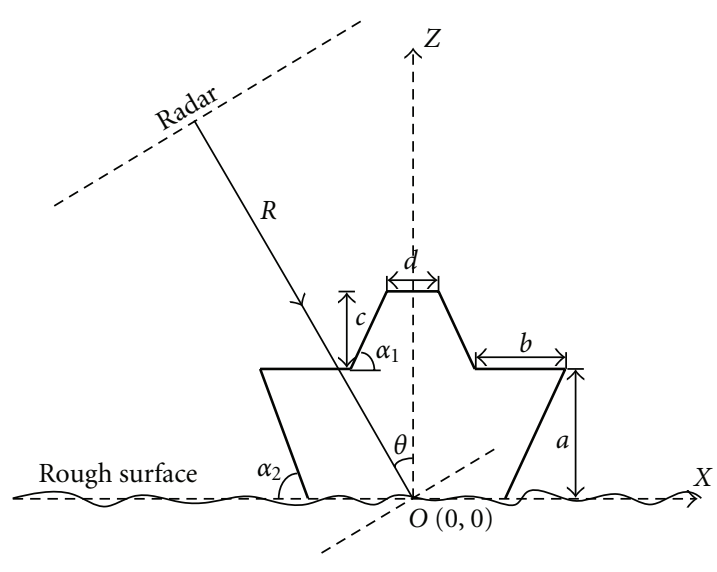

FIgUre 1: The model of a ship on sea surface.

besides the strong coupling interactions with the sea. The multipath coupling will be strongly affected when the sea state changes. Therefore, the multipath imaging mechanisms between the ship and sea are much more complicated and have been remained for further study in this paper.

In this paper, the $1 \mathrm{D}$ HRRPs of a ship on the PEC sea surface are simulated via the same numerical method as in [9]. The scattered field data is sampled from $100 \mathrm{MHz}$ to $700 \mathrm{MHz}$. Although the sampled frequency is not very high, the electrical size of the ship chosen in this study is large enough. Besides, the bandwidth is also large enough to achieve a high range resolution. The characteristics of the high resolution range profiles, especially the multipath returns caused by the complicated coupling interactions are analyzed by means of ray theory prediction and numerical extraction of the coupling currents. A discussion about the differences of the range profile locations between ray theory prediction and surface current simulation is further presented. Besides, the influence of different wind speeds for the rough sea surface to the radar signatures has been examined and discussed through numerical examples.

The remainder of the paper is organized as follows. In Section 2, we briefly describe the problem of ship on the rough sea surface. In Section 3, the $1 \mathrm{D}$ range profiles are provided and analyzed. Finally, a conclusion is given in Section 4.

\section{Statement of the Problem}

Figure 1 illustrates the composite model of a 2 D ship located on a rough sea surface with a Pierson-Morkowitz spectrum [9]. A TE-polarized tapered wave is incident with angle $\theta$, and the backscattered far field is received by the radar. Suppose $R$ is the distance between the radar and the reference point $O$. To obtain the exact scattered field in the frequency domain, the problem is reduced to finding the induced surface currents by the numerical method [9].
Here, we consider the $2 \mathrm{D}$ simple ship with parameters as follows:

$$
\begin{gathered}
a=20 \mathrm{~m}, \quad b=16 \mathrm{~m}, \quad c=12 \mathrm{~m}, \quad d=8 \mathrm{~m}, \\
\alpha_{1}=25^{\circ}, \quad \alpha_{2}=20^{\circ} .
\end{gathered}
$$

The frequency response of the scattering system is simulated using a stepped frequency waveform (SFW) [10], which is produced by linearly sampling the desired bandwidth $B$ at specific frequencies. The parameters of the SFW are appropriately designed considering the size of the composite model. The scattered field is sampled from $100 \mathrm{MHz}$ to $700 \mathrm{MHz}(8.53 \leq D / \lambda \leq 59.72$, where $\lambda$ is the wavelength $D$ is the diameter of the smallest circle containing the whole ship); thus, the bandwidth is $B=600 \mathrm{MHz}$, and the high range resolution is $\Delta R=c / 2 B=0.25 \mathrm{~m}$. A frequency step $\Delta f=2 \mathrm{MHz}$ is considered to obtain the sufficient unambiguous range $R_{u}=c / 2 \Delta f=75 \mathrm{~m}$. By performing an inverse discrete Fourier transform (IDFT) on the backscattered field data in frequency domain, the time domain response is provided for the wide-band signal. Accordingly, the $1 \mathrm{D}$ range profiles of the ship on the rough sea surface is obtained, since the time delay of the signal is associated with the positions of the scattering centers projected on the line of the radar sight.

With the help of the numerical simulation of surface currents, the contribution of the coupling scatterings to the signatures could be extracted and analyzed by separating the coupling currents on the ship and sea surface. The total received field could be seen as a coherent summation of the radiations from four current components, namely $\mathbf{J}_{t 0}, \mathbf{J}_{s 0}$, $\mathbf{J}_{t d}$, and $\mathbf{J}_{t d}$. Here, $\mathbf{J}_{t 0}$ and $\mathbf{J}_{s 0}$ represent the induced surface currents on the ship and sea when illuminated in free space, respectively. The contribution of $\mathbf{J}_{t 0}$ corresponds to the direct back-scattered return of the isolated ship without interaction with the sea surface. Similarly, $\mathbf{J}_{s 0}$ corresponds to the direct back-scattered signal from the sea surface individually. For planar sea surface, it could be predicted that there is no back-scattered return due to the total specular scattering. For the rough sea surface, there is the possibility of strong nonspecular scattering complicating the radar returns.

Specifically, the ship-sea coupling interactions are caused by the two interacting current terms $\mathbf{J}_{t d}$ and $\mathbf{J}_{s d}$. Physically, the first term corresponds to the radar echoes returned back from the ship and the other referred to the signal finally travels back from the sea surface. The coupling between ship and sea will give rise to multipaths of different interaction orders. It is of interest to see how the strong complicated interactions affect the scattering features of the ship on the sea surface, which is quite different from the case when the target is far away above the sea surface. By performing an IDFT on the extracted coupling terms, the range profiles due to different terms could be separated and analyzed to help a better understanding of the coupling behaviors and received signatures. Meanwhile, the prediction of the multipath locations expected by the ray theory helps to identify the important scattering mechanisms. 


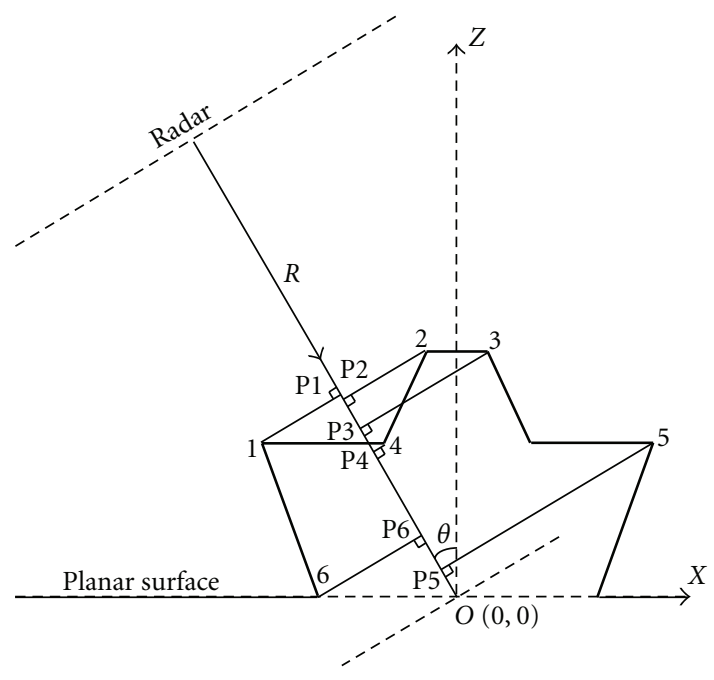

FIGURE 2: Individual scattering centers of the ship.

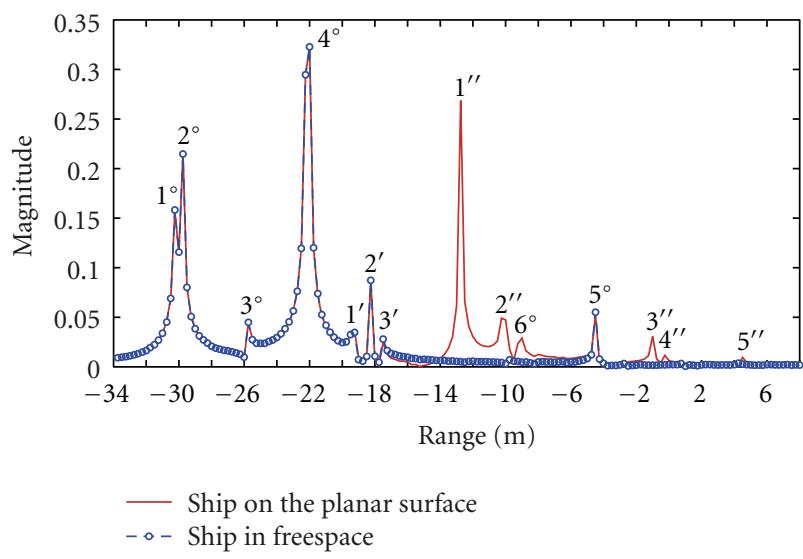

FIGURE 3: Range profiles of the ship on planar sea surface.

\section{Numerical Results and Discussions}

It is expected that the range profiles will provide information about the position and scattering strength of the scattering centers along the range direction. The incident direction is chosen as the elevation angle $\theta=30^{\circ}$. Figure 2 illustrates the five individual scattering centers of the ship contributed by its edges. According to the ray theory, the locations of the five scattering centers projected on the incident direction could be predicted and marked as P1, P2, P3, P4, P5.

Firstly, the $1 \mathrm{D}$ HRRPs of the ship on the planar surface is shown in Figure 3 (solid line) and compared with the case of the ship in free space. The reference point $O$ in Figure 2 corresponds to a range location of zero in Figure 3. It is found that the locations of the simulated range profiles $1^{\circ}, 2^{\circ}, 3^{\circ}$, $4^{\circ}, 5^{\circ}$ match well with the ray theory predictions of the ship's five individual scattering centers. The detailed comparison of the range locations made between the numerical simulation and the ray theory expectation is demonstrated in Table 1.

The wideband signatures are strongly related with the complex geometry of the ship. It is also expected that
TABLE 1: Comparison of simulated and expected range locations.

\begin{tabular}{lcccc}
\hline Peak & Current terms & $\begin{array}{c}\text { Current } \\
\text { simulation } \\
\text { Simulated }(\mathrm{m})\end{array}$ & $\begin{array}{c}\text { Ray theory } \\
\text { prediction } \\
\text { Expected }(\mathrm{m})\end{array}$ & Error \\
\hline $1^{\circ}$ & $\mathbf{J}_{t 0}$ & -30.15 & -30.1128 & 0.0372 \\
$2^{\circ}$ & $\mathbf{J}_{t 0}$ & -29.7 & -29.7128 & -0.0128 \\
$3^{\circ}$ & $\mathbf{J}_{t 0}$ & -25.65 & -25.7128 & -0.0628 \\
$4^{\circ}$ & $\mathbf{J}_{t 0}$ & -22.05 & -22.1128 & -0.0628 \\
$5^{\circ}$ & $\mathbf{J}_{t 0}$ & -4.5 & -4.5227 & -0.0227 \\
$6^{\circ}$ & $\mathbf{J}_{t d} \mathbf{J}_{s d}$ & -9.15 & -9.1581 & -0.0081 \\
$1^{\prime}$ & $\mathbf{J}_{t 0}$ & -19.35 & -19.3205 & 0.0295 \\
$2^{\prime}$ & $\mathbf{J}_{t 0}$ & -18.3 & -18.2399 & 0.0601 \\
$3^{\prime}$ & $\mathbf{J}_{t 0}$ & -17.55 & -17.5627 & -0.0127 \\
$1^{\prime \prime}$ & $\mathbf{J}_{t d} \mathbf{J}_{s d}$ & -12.75 & -12.7978 & -0.0478 \\
$2^{\prime \prime}$ & $\mathbf{J}_{t d}$ & -10.05 & -10.1184 & 0.0684 \\
$3^{\prime \prime}$ & $\mathbf{J}_{t d} \mathbf{J}_{s d}$ & -0.9 & -0.9194 & -0.0194 \\
$4^{\prime \prime}$ & $\mathbf{J}_{t d} \mathbf{J}_{s d}$ & -0.15 & -0.2422 & 0.0922 \\
$5^{\prime \prime}$ & $\mathbf{J}_{s d}$ & 4.5 & 4.5227 & 0.0227 \\
\hline
\end{tabular}

the corner reflector of the deck house will produce multipath returns. The detail ray paths illustrated in Figures 4(a), 4(b), and $4(\mathrm{c})$ correspond to the range profile peaks $1^{\prime}, 2^{\prime}, 3^{\prime}$ shown in Figure 3.

Besides, the strong coupling interactions between the ship and the bottom surface are expected to produce equivalent range profiles. It is found that the location of peak $6^{\circ}$ corresponds to the corner edge formed by the ship hull and the bottom surface, as illustrated in Figure 2. It could be expected that the delayed peaks $1^{\prime \prime}, 2^{\prime \prime}, 3^{\prime \prime}, 4^{\prime \prime}, 5^{\prime \prime}$ in Figure 3 are generated by the multipath returns between ship and sea. The peak $1^{\prime \prime}$ is the first-order range profile caused by the double bounce between the left edge of the hull and the planar sea surface. Peaks $2^{\prime \prime}, 3^{\prime \prime}, 4^{\prime \prime}, 5^{\prime \prime}$ are contributed by higher order coupling interactions. The corresponding ray paths for the multipath returns are clearly illustrated in Figure 4(d) and Figure 5.

Figure 6 demonstrates the contribution of the coupling current terms to the equivalent range profiles. It is discovered that both the two current terms contribute to peak $1^{\prime \prime}, 3^{\prime \prime}, 4^{\prime \prime}$, and $6^{\circ}$. This could be physically explained as the ray paths are reversible and the multipath wave could return either from the ship or the sea surface. It should be noted that the interference of the two current terms generates not only strengthened peaks $\left(1^{\prime \prime}, 3^{\prime \prime}, 4^{\prime \prime}\right)$ but also a reduced peak like $6^{\circ}$. Besides, only one current term contributes to peak $2^{\prime \prime}\left(\mathbf{J}_{t d}\right)$ and peak $5^{\prime \prime}\left(\mathbf{J}_{s d}\right)$ due to the irreversible ray paths.

The ray theory predictions based on the scattering center approximation include the dominant high frequency scattering mechanisms, such as edge diffraction or specular refection. The agreement of the peak locations between the ray theory prediction and the surface current simulation seems good in Table 1. However, the difference of the locations between the two methods still exists and could be observed from Table 1. The calculation of the radiation from the accurate surface currents yields the real phase 


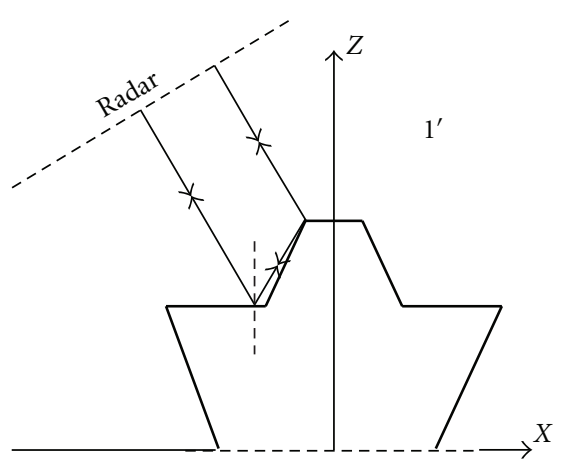

(a)

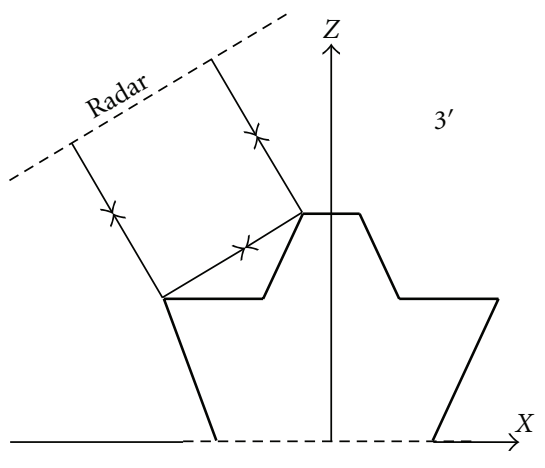

(c)

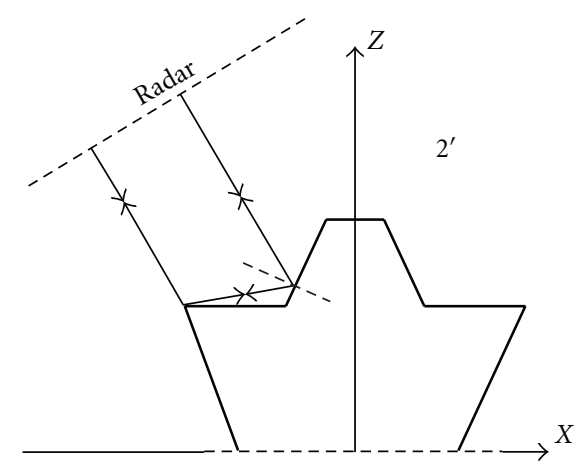

(b)

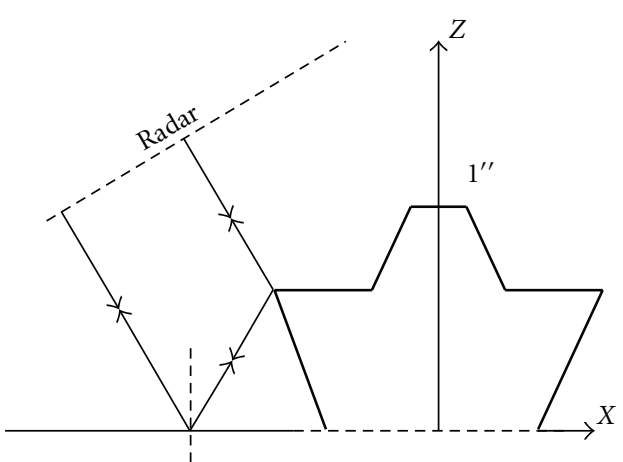

(d)

FIgURE 4: Multipath returns (I).

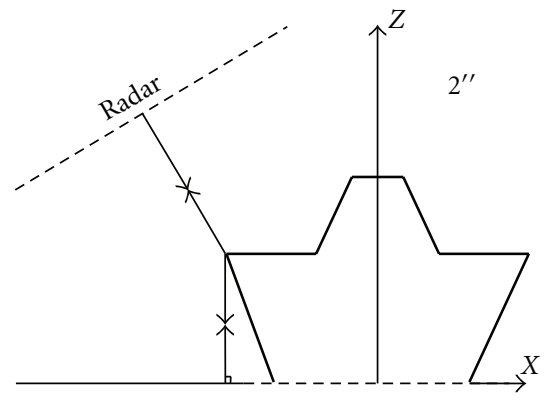

(a)

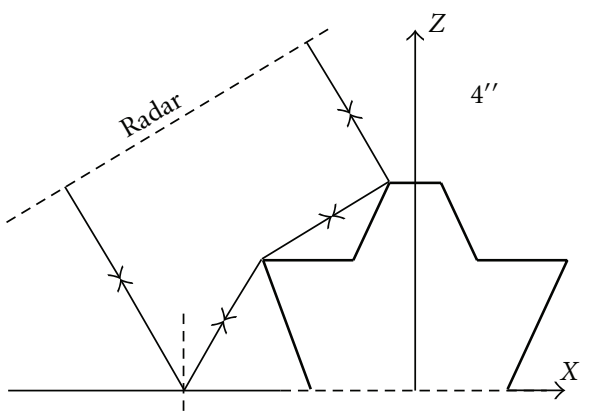

(c)

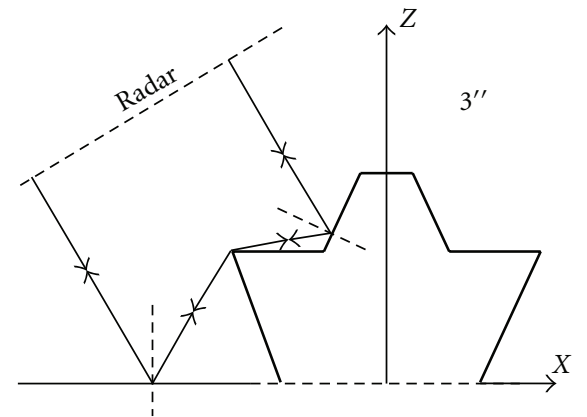

(b)

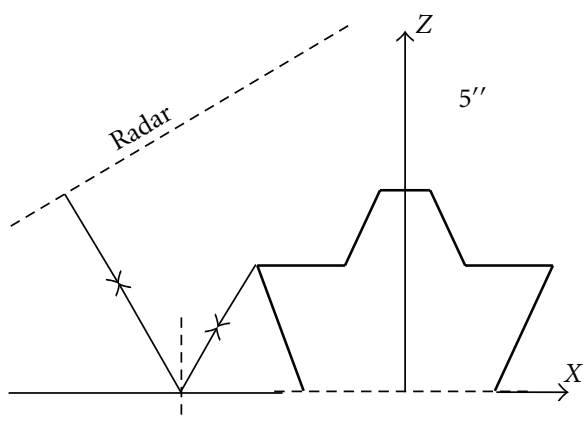

(d)

FIgURE 5: Multipath returns (II). 


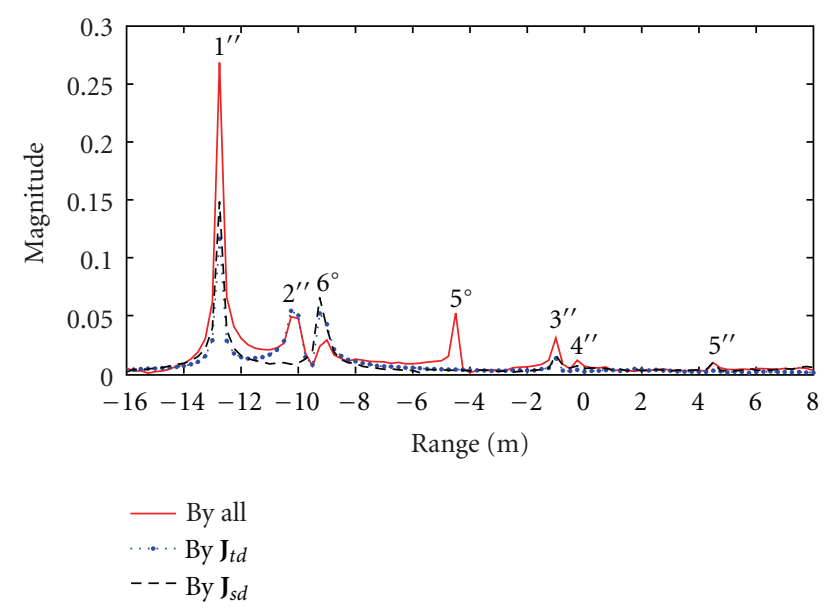

FIGURE 6: Range profiles contributed by coupling current terms.

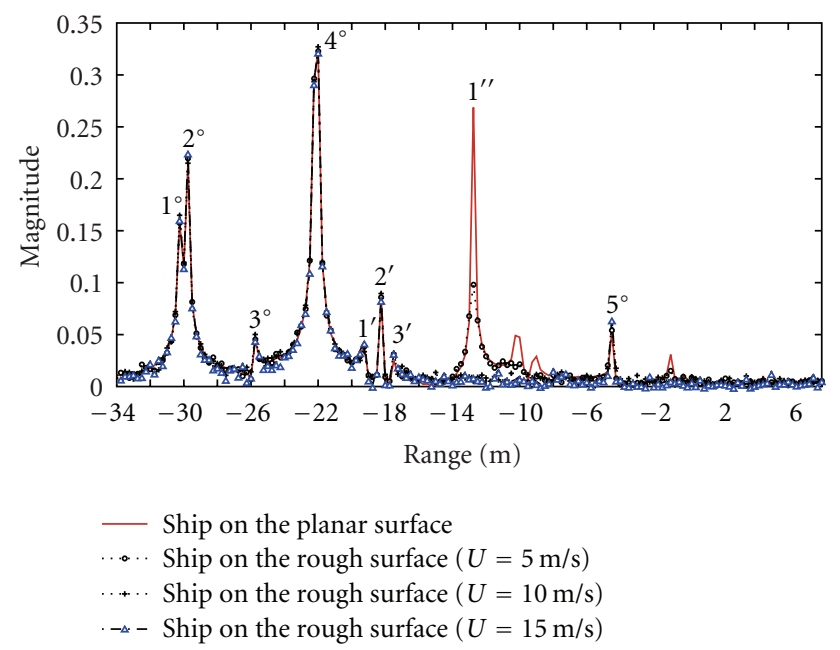

FIGURE 7: Range profiles of the ship on sea with 100 realizations.

results of the electromagnetic field. However, the ray theory predictions of the phase for the radar return deviate from the real value and do not represent the true location. The deviations of the ray theory predictions to the accurate simulations are inevitable, and the errors listed in Table 1 are analyzed in the following.

As shown in Figure 2, edge 1 and edge 5 that are taken as the individual scattering centers have the similar geometry structure. However, it should be noted that the induced surface currents are not the same on each edge surface of the ship, which also means that their scattering behavior is different with respect to the aspect direction. Therefore, the deviation of the ray theory predicted locations $\left(1^{\circ}\right.$ and $\left.5^{\circ}\right)$ for the corresponding edges 1 and 5 appears different, which could be observed in the table. It could be further observed that the ray theory prediction leads to both minus and plus deviations (errors). For the multipath return shown in
Figures 4 and 5, complex coupling mechanisms are involved, such as edge-face interaction and edge-edge interaction. As for the edge-face interactions, the face could be either the bottom surface or the finite flat surface of the ship. So for the predicted location of the multipath return, the accumulated error along the path is very complicated due to multitime couplings. The final deviation may be enlarged or decreased because of the minus or plus errors brought in by the scattering center approximation.

The different order interactions between the ship and the planar surface give rise to different order equivalent range profiles. The amplitude of the first-order range profile peak $1^{\prime \prime}$ is the largest of all due to the strong corner reflector caused by the left edge of the hull and the planar sea surface. It is obvious in Figure 3 that the higher-order range profiles $2^{\prime \prime}, 3^{\prime \prime}, 4^{\prime \prime}, 5^{\prime \prime}$ are detected with much lower amplitude due to the weak scattering mechanisms and the attenuation of the propagation. Of the four higher order range profiles, the scattering mechanisms involved are edge diffractions and specular reflections. Since the specular-reflected energy remains almost unchanged, the strength of the higher-order range profiles is mainly determined by the diffraction of the edges. The strength of the diffraction fields is related to the wedge angle of the structure and the incident angle with respect to the wedge faces. It could be concluded from Figures 3 and 5 that the diffraction happened in the multiple scattering of range profile $2^{\prime \prime}$ is much stronger than that happened in the scattering of the other range profiles.

Considering the real marine environment, the range profiles of the ship on a wind driven sea surface are demonstrated in Figure 7. The wind speed is chosen as $U=$ $5 \mathrm{~m} / \mathrm{s}, U=10 \mathrm{~m} / \mathrm{s}$, and $U=15 \mathrm{~m} / \mathrm{s}$. In order to avoid the randomness of the rough surface for the Monte Carlo simulation, 100 realizations are considered. It could be noted that the different sea states have very little affection on the ship's individual scattering centers. For the largest wind speed $U=15 \mathrm{~m} / \mathrm{s}$, the peak value of the individual scattering centers is observed with a very small change compared with the planar surface case $(U=0 \mathrm{~m} / \mathrm{s})$.

However, compared with the planar surface case, the coupling interactions between the ship and sea are decreased significantly because of the rough surface diffuse scattering. For $U=5 \mathrm{~m} / \mathrm{s}$, the comparison suggests that the peak of the higher interactions can no longer be identified while the first-order peak $1^{\prime \prime}$ has a sharp decrease. As the wind speed increases to more than $10 \mathrm{~m} / \mathrm{s}$, even the first-order peak becomes too small to be distinguished from the random scattering.

\section{Conclusion}

In this paper, a range profile analysis is implemented for a simplified 2 D ship on wind-driven sea surface. A detailed study of the coupling imaging mechanisms for range profile characteristics is performed. The investigation of the coupling multipath provides guidelines for automatic target recognition. Therefore, it is our future task to analyze the $3 \mathrm{D}$ problems which are of more practical importance. 


\section{Acknowledgments}

This work was supported by the National Natural Science Foundation of China (Grant no. 61001059), the China Postdoctoral Science Foundation, and the Fundamental Research Funds for the Central Universities.

\section{References}

[1] E. A. Shtager, "An estimation of sea surface influence on radar reflectivity of ships," IEEE Transactions on Antennas and Propagation, vol. 47, no. 10, pp. 1623-1627, 1999.

[2] R. J. Burkholder, M. R. Pino, and F. Obelleiro, "A Monte Carlo study of the rough-sea-surface influence on the radar scattering from two-dimensional ships," IEEE Antennas and Propagation Magazine, vol. 43, no. 2, pp. 26-33, 2001.

[3] K. Jamil and R. J. Burkholder, "Radar scattering from a rolling target floating on a time-evolving rough sea surface," IEEE Transactions on Geoscience and Remote Sensing, vol. 44, no. 11, pp. 3330-3337, 2006.

[4] Y. Liang, L. Guo, and Z. S. Wu, “The EPILE combined with the generalized-FBM for analyzing the scattering from targets above and on a rough surface," IEEE Antennas and Wireless Propagation Letters, vol. 9, pp. 809-813, 2010.

[5] M. R. Pino, R. J. Burkholder, and F. Obelleiro, "Spectral acceleration of the generalized forward-backward method," IEEE Transactions on Antennas and Propagation, vol. 50, no. 6, pp. 785-797, 2002.

[6] F. Xu and Y. Q. Jin, "Bidirectional analytic ray tracing for fast computation of composite scattering from electric-large target over a randomly rough surface," IEEE Transactions on Antennas and Propagation, vol. 57, no. 5, pp. 1495-1505, 2009.

[7] S. Y. He and G. Q. Zhu, "A hybrid MM-PO method combining UV technique for scattering from two-dimensional target above a rough," Microwave and Optical Technology Letters, vol. 49, no. 12, pp. 2957-2960, 2007.

[8] F. S. Deng, S. Y. He, H. T. Chen, W. D. Hu, W. X. Yu, and G. Q. Zhu, "Numerical simulation of vector wave scattering from the target and rough surface composite model with 3-D multilevel UV method," IEEE Transactions on Antennas and Propagation, vol. 58, no. 5, pp. 1625-1634, 2010.

[9] S. Y. He, F. S. Deng, H. T. Chen, W. D. Hu, W. X. Yu, and G. Q. $\mathrm{Zhu}$, "Range profile analysis of the 2-D target above a rough surface based on the electromagnetic numerical simulation," IEEE Transactions on Antennas and Propagation, vol. 57, no. 10, pp. 3258-3263, 2009.

[10] H. Y. Chen, Y. X. Liu, W. D. Jiang, and G. R. Guo, "A new approach for synthesizing the range profile of moving targets via stepped-frequency waveforms," IEEE Geoscience and Remote Sensing Letters, vol. 3, no. 3, pp. 406-409, 2006. 

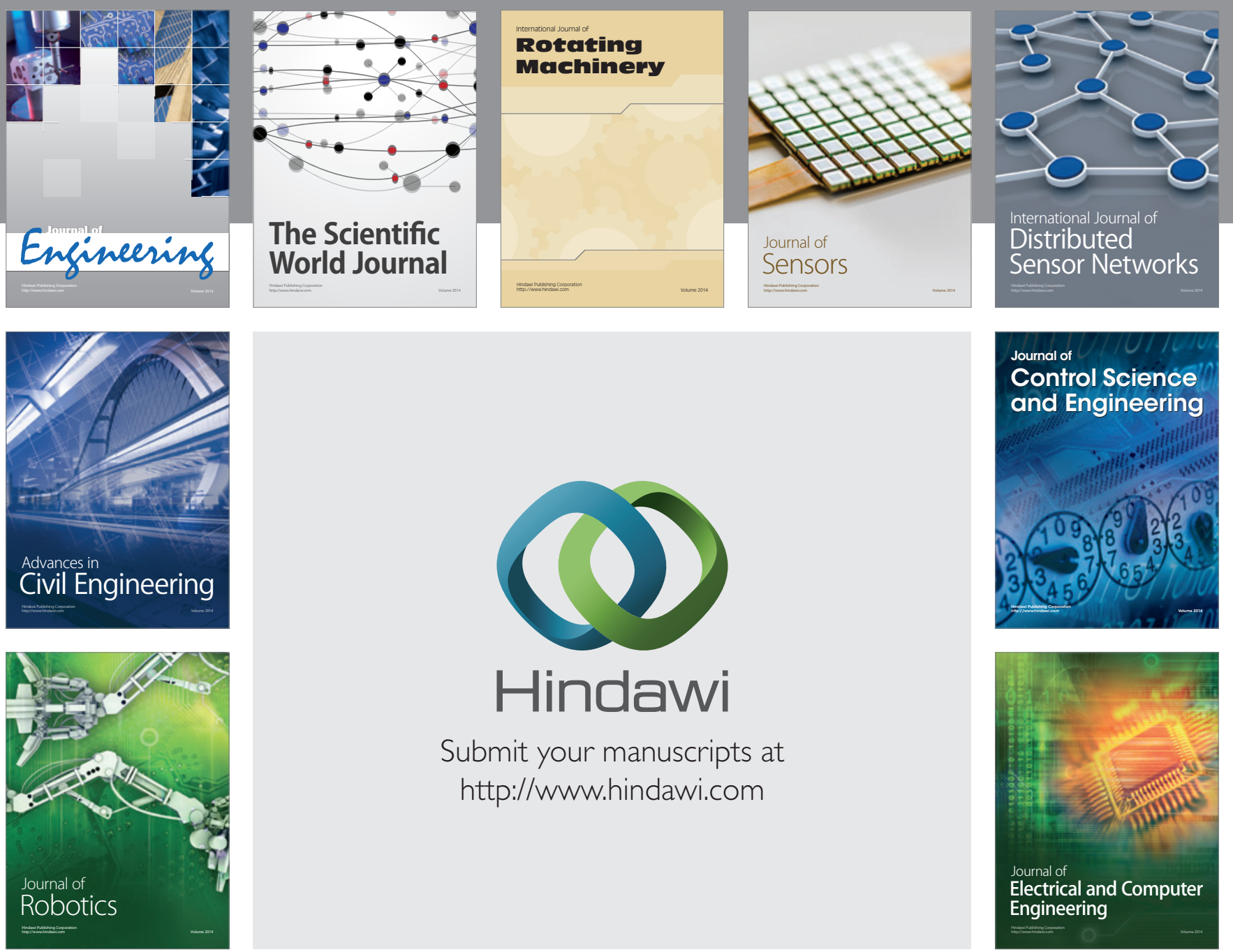

Submit your manuscripts at

http://www.hindawi.com
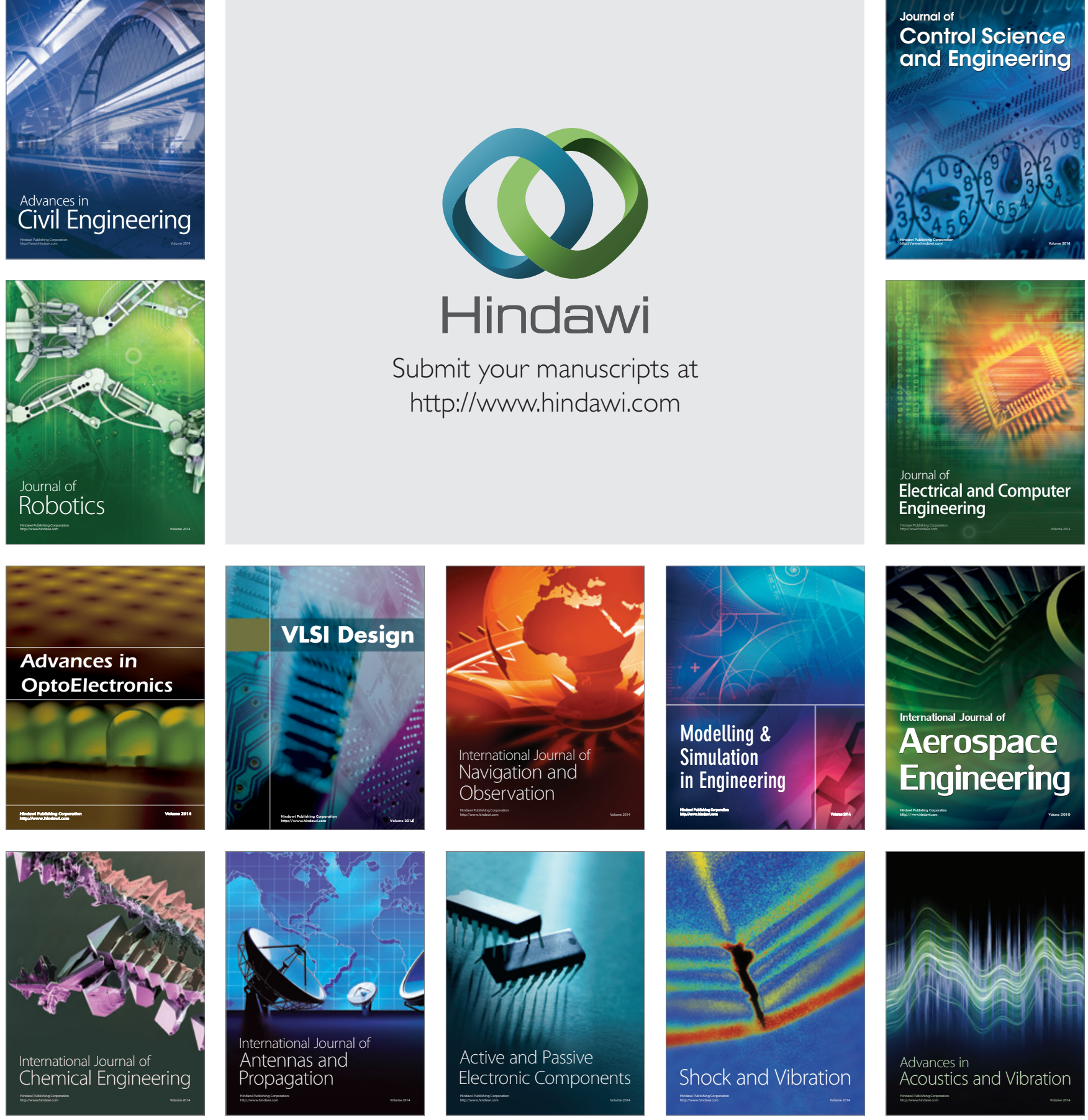